\section{Atopische Dermatitis: Codierung nach ICD-9 kann in die Irre führen}

Hsu DY et al. Validation of International Classification of Disease Ninth Revision codes for atopic dermatitis. Allergy 2017; 72: 1091 - 1095

Um Epidemiologie, Begleiterkrankungen und Ausmaß einer atopischen Dermatitis zu untersuchen, werden oft großen Datenbanken herangezogen. Die beste Methode, daraus die betroffenen Patienten zu identifizieren, ist bislang aber noch unklar: Häufig wird der ICD-9-Code verwendet, aber ist dieses Vorgehen valide? Das habe nun Mediziner der Northwestern University in Chicago geprüft.

Hsu et al. haben dazu die elektronischen Krankenakten der Universitätsklinik herangezogen und Daten für die Codierungen 691.8 (atopische Dermatitis) und 692.9 (Ekzem oder chronische Kontaktdermatitis) extrahiert. Dann prüften sie, inwieweit die Diagnose „atopische Dermatitis" anhand der Kriterien von Hanifin und Rajka sowie den Kriterien der United Kingdom Working Party (UKWP) erfüllt waren.

Dabei galt die Diagnose als gesichert, wenn mindestens 3 Haupt- und 3 Nebenkriterien nach Hanifi und Rajka erfüllt waren. Hauptkriterien umfassen dabei Pruritus, typisches Aussehen und typische Lokalisation der Hautveränderungen, chronisch rezidivierenden Verlauf der Erkrankung und atopische Eigenund/oder Familienanamnese. Nebenkriterien sind beispielsweise DennieMorgan-Falten, Herthoge-Zeichen oder Ohr-Rhagaden. Eine wahrscheinliche Diagnose lag bei Erfüllen von mindestens 2 Haupt- und 2 Nebenkriterien vor.

Die Kriterien der UKWP fordern für eine gesicherte Diagnose als unbedingt notwendig eine mit Juckreiz verbundene Hauterkrankung in den zurückliegenden 12 Monaten plus mindestens 3 Begleitsymptome, z.B. allgemeine Hauttrockenheit oder Lokalisation der Befunde über den Beugeseiten der Gelenke. Bei nur 2 Begleitsymptomen galt die Diagnose als wahrscheinlich.

Die Forscher überprüften die Zuverlässigkeit der Codierungen anhand der genannten Kriterien als Referenz und untersuchten außerdem Überlappungen des Codes 691.8 mit dem Code 692.9.

Die Auswertung ergab zunächst mehr als 40000 Patienten mit dem Diagnosecode 691.8 und mehr als 2000 Patienten mit dem Diagnosecode 692.9. Daraus suchten die Mediziner dann eine Zufallsstichprobe von 519 Patienten mit dem Code 691.8 und 253 mit dem Code 692.9 heraus und prüften die Diagnose anhand der Akten nach den oben genannten Kriterien. Dabei fanden sie zunächst eine starke Überlappung der beiden Codierungen: Bei 198 Patienten (34,8\%) waren mindestens 1-mal beide Codierungen verwendet worden, bei 149 Patienten war das sogar mehrfach der Fall. In den Akten zeigte sich dann, dass die Diagnose einer atopischen Dermatitis nach den Hanifin-Rajka-Kriterien bzw. den UKWPKriterien

- bei 29,9\% bzw. 30,8\% der Patienten mit mindestens 1-maliger Codierung 691.8 bestand, sowie

- bei 33,7\% bzw. 32,2\% Patienten mit mindestens 1-maliger Codierung 692.9.

Daraus errechnete sich ein positiver Vorhersagewert der Diagnose 691.8 für das tatsächliche Vorliegen einer atopischen Dermatitis von 31,3\%, und dieser Wert nahm auch nicht zu, wenn nur Patienten mit mehrmaliger 691.8-Codierung berücksichtigt wurden. $\mathrm{Er}$ verbesserte sich allerdings deutlich, auf 46,6\% bzw. 47,1\% (Hanifin-Rajka- bzw. UKWP-Kriterien), wenn nur Patienten einbezogen wurden, bei denen zusätzlich ein Asthma oder eine allergische Rhinitis bestand. Wurde außerdem eine Lebensmittelallergie gefordert, waren es 76,3\% bzw. $65,8 \%$. Für den Diagnosecode 692.9 lagen die Verhältnisse ähnlich.

Schließlich untersuchten die Wissenschaftler noch die Subgruppe der stationär behandelten Patienten: Ein Code 691.8 lag bei 10 Patienten vor, davon erfüllten 9 die Hanifin-RajkaKriterien (90\%) und 10 die UKWP-Kriterien (100\%). Nur bei 1 stationären Patienten war eine 692.9-Diagnose angegeben, der nach allen Kriterien vermutlich keine atopische Dermatitis aufwies.

FAZIT

Verlässt man sich bei wissenschaftlichen Untersuchungen zur atopischen Dermatitis bei ambulanten Patienten ausschließlich auf die Codierung 691.8 , ist das Risiko von Fehlinterpretationen hoch, so die Autoren. Auch mit dem neueren ICD-10-Code, der in den USA seit 2013 und in Europa seit mehr als 10 Jahren eingesetzt wird, scheinen systematische Irrtümer immer noch möglich. Zusätzliche Diagnosen wie Asthma und Lebensmittelallergie verbessern die Aussagekraft, wenn auch auf Kosten der Sensitivität.

\section{Dr. Elke Ruchalla, Bad Dürrheim}

